\title{
Impact of 5a-Reductase Inhibitors and a-Blockers for Benign Prostatic Hyperplasia on Prostate Cancer Incidence and Mortality
}

\author{
Dr. Maria I. Van Rompay, PhDa, J. Curtis Nickel, MD' ${ }^{b}$, Gayatri Ranganathan, MS ${ }^{a}$, Philip W. \\ Kantoff, MD ${ }^{c}$, Keith R. Solomon, $\mathrm{PhD}^{\mathrm{d}}$, Jennifer L. Lund, $\mathrm{PhD}^{\mathrm{e}}$, and John B. McKinlay, \\ PhD ${ }^{a, f}$ \\ aHealthCore/NERI, 480 Pleasant Street, Watertown, MA 02472 USA \\ ${ }^{b}$ Queen's University and Kingston General Hospital, Kingston, Ontario, Canada \\ cMemorial Sloan Kettering Cancer Center and Weill Cornell Medical College, New York, NY \\ 10065 USA \\ dDepartment of Orthopaedic Surgery, Harvard Medical School, and the Departments of \\ Orthopaedic Surgery and Urology, Boston Children's Hospital, Boston, MA 02115 USA. Current \\ address: Applied Photophysics, 100 Cummings Center, Suite 440C, Beverly, MA 01915 USA \\ eDepartment of Epidemiology, University of North Carolina at Chapel Hill, NC USA \\ fMassachusetts General Hospital, Harvard Medical School, Boston, MA 02114 USA
}

\section{Abstract}

Objective: To investigate the use of 5a-reductase inhibitors (5ARIs) and a-blockers among men with benign prostatic hyperplasia (BPH) in relation to prostate cancer (PC) incidence, severity, and mortality.

Patients and Methods: A retrospective 20-year cohort study in Saskatchewan men aged 40-89 years with a BPH-coded medical claim between 1995 and 2014 was conducted. Cox proportional hazards regression was used to compare incidence of a PC diagnosis, metastatic PC, Gleason score 8-10 PC, and PC mortality among 5ARI users $(n=4,571)$, $a$-blocker users $(n=7,764)$, and nonusers $(\mathrm{n}=11,677)$.

Results: In comparison with both non-users and a-blocker users, 5ARI users had approximately $40 \%$ lower risk of a PC diagnosis ( $11.0 \%$ and $11.4 \%$ vs. $5.8 \%$, respectively); a-blocker users had $11 \%$ lower risk of a PC diagnosis compared with non-users. Overall, there was no significant increase in metastatic PC or PC mortality among 5ARI or a-blocker users (metastatic PC: $0.8 \%$ and $1.5 \%$ vs. $1.4 \%$ [non-users]; PC mortality: $1.2 \%$ and $2.4 \%$ vs. $2.2 \%$ [non-users], respectively, $\mathrm{P}>0.05$ for both drugs) but there was approximately $30 \%$ higher risk of Gleason score $8-10$ cancer

Corresponding Author: Maria I. Van Rompay, PhD; HealthCore/NERI, 480 Pleasant Street, Suite A100, Watertown, MA 02472 USA., Tel: +1 (617) 972-3281; Fax: +1 (617) 673-9547; mvanrompay@ neriscience.com.

Conflict of Interest

None declared. 
(1.4\% and $1.8 \%$ vs. $2.0 \%$ [non-users], aHR: $1.37,95 \% \mathrm{CI}: 1.03-1.82, \mathrm{P}=0.03$ and aHR: $1.28,95 \%$ CI: $1.03-1.59, \mathrm{P}=0.02$, respectively) compared with non-users.

Conclusion: 5ARI use was associated with lower risk of a PC diagnosis, regardless of comparison group. Risk of high-grade PC was higher among both 5ARI users and a-blocker users compared with non-users; however, this did not translate into higher risk of PC mortality.

\section{Keywords}

prostatic hyperplasia; benign; dutasteride; finasteride; adrenergic alpha-antagonists; prostatic neoplasms; pharmacoepidemiology

\section{Introduction}

Mortality from prostate cancer (PC) (20.7 per 100,000 men per year) is markedly lower than incidence (age-adjusted rate of 129 per 100,000) [1]. Because of its common occurrence, and disease attributes such as long latency and late age at diagnosis, PC may be an ideal target for chemoprevention [2]. Landmark randomized placebo-controlled trials examining 5a-reductase inhibitors (5ARIs), finasteride and dutasteride, have reported 25\% lower incidence of diagnosed PC among both healthy [3, 4] and high-risk [5, 6] men randomized to 5ARIs, a risk reduction substantiated by a 2010 systematic review [7]. However, PC chemoprevention by 5ARIs is not currently recommended due to evidence from the Reduction by Dutasteride of Prostate Cancer Events (REDUCE) and Prostate Cancer Prevention Trial (PCPT) studies that 5ARI users had a higher incidence of high-grade Gleason score $8-10$ cancers $[1,3-5,8]$.

For PC outcomes in BPH populations, use of dutasteride with or without tamsulosin was associated with a $40 \%$ relative risk reduction of a PC diagnosis in the Combination of Avodart and Tamsulosin (CombAT) trial [9] and a 34\% risk reduction in a meta-analysis [10]. Observational data investigating use of an a-blocker alone have shown both no association with PC risk in comparison with non-users [11,12] as well as 3.6 times the risk of PC compared with general population controls [13]. In regard to Gleason score 8-10 cancer and PC metastases at diagnosis, data on pre-diagnostic 5ARI use suggest no difference in risk between 5ARI users and non-users in a UK population [14]. A lack of association in relation to PC mortality for 5ARI therapy is relatively consistent $[4,11,14$ 16], except for a Danish population study in which both 5ARIs and a-blockers were associated with increased risk in men with BPH compared with general population controls [13]. In the Finnish Prostate Cancer Screening Trial, men who used a-blockers either before or after a PC diagnosis had increased risk of PC mortality; however, this increased risk diminished with longer-term a-blocker use [16].

The overall objective of our study was to investigate the use of these prescribed drugs in routine clinical practice and their potential to impact the risk of PC incidence, severity, and mortality in a large community-dwelling population-based sample. Specific objectives were to use a cohort study design to estimate the risk of incident PC, metastatic PC, and PC mortality among 5ARI users, a-blocker users, and non-users in men with a physician claim reporting BPH in a large community-based sample with 10-20 years of follow-up time. 


\section{Patients and Methods \\ Data Source and Population}

The Saskatchewan Ministry of Health compiled de-identified data for 249,986 men aged $\geq 40$ years covered by Saskatchewan Health (SH) and eligible for provincial outpatient prescription drug benefits between January 1, 1995 and December 31, 2014. Based on predefined criteria, the dataset included a subset of the men's outpatient prescription drug, medical services, and health insurance registration records, as well as cancer records from the Saskatchewan Cancer Agency (SCA) cancer registry. The study was approved by the institutional review board of HealthCore/NERI, Watertown, MA, and the University of Saskatchewan Biomedical Research Ethics Board.

\section{Drug Exposure Groups}

We identified male new users of finasteride ( $5 \mathrm{mg}$ daily), dutasteride ( $0.5 \mathrm{mg}$ daily), and/or a-blockers, including alfuzosin, doxazosin, prazosin, tamsulosin or terazosin. Index date was defined as dispensing date of the first prescription on or after the man's $40^{\text {th }}$ birthday. For each finasteride and dutasteride user, at least two non-users were selected and matched on age (up to \pm 2 years). Non-users were assigned the same index date as their matched users, and users were eligible to serve as non-users prior to their first index prescription. Men with a history of cancer prior to the index date were excluded, as well as those with $<365$ days of SH coverage prior to index, as the first prescription could not reliably be determined. For the present analyses, exposure groups were defined as having ever used the drug, as of the index date, and a-blocker users had a BPH medical claim within 12 months prior to index $(\mathrm{n}=7,764), 5 \mathrm{ARI}$ users had a BPH medical claim within 12 months pre-index through 3 months post-index $(n=4,571)$ [17], and non-users had a BPH medical claim anytime during follow-up $(\mathrm{n}=11,677)$.

\section{Outcomes}

Incident PCs were identified from SCA registry data using first occurrence date of ICD-O-3 code C61.9 for primary prostate carcinoma, focusing on adenocarcinomas. Information on PC mortality was obtained from SCA registry data on cause of death. To define more advanced PC, evidence of metastases at diagnosis was used. While the Gleason classification system has previously been widely used, we did not rely upon these data due to considerable variability in assigning Gleason scores among pathologists and over time [18-20]. However, for comparison with previous research, we present results of secondary analyses using Gleason score 8-10 cancers (versus lower grades). Reasons for missing Gleason score data include changes in the coding of cancer severity data over time by the SCA. Specific data on screening methods and/or diagnostic medical procedures associated with PC diagnoses evolved over time during the 20-year follow-up period, without sufficient consistency to enable proper characterization. Nonetheless, the study's outcomes derive from valid, concrete parameters documented in the SCA database, including PC diagnosis, metastatic PC, mortality, and, for a subpopulation with Gleason score data, tumor grade. 


\section{Covariates}

Covariates included age at index date, index (calendar) year, baseline use of lipid-lowering medications, and baseline diagnosis of cardiometabolic conditions including diabetes, hypertension, heart disease, and hyperlipidemia; "baseline" was defined as within 12 months prior to index through index date. Physician services records provided data on certain medical diagnoses of interest; however, only one diagnosis was reported for a medical visit. Further, some physicians in Saskatchewan were on alternative payment plans with the health region or province and may have prescribed a drug without record of a physician visit. For analysis, index year was initially categorized into quartiles, but due to small numbers in the first two categories, tertiles were used: 1995-2004 (reference), 2005-2009, and 2010-2014.

\section{Statistical Analyses}

Demographic and clinical characteristics of new users of 5ARIs, a-blockers, and non-users of both drugs with a BPH-coded medical claim were compared. A modified case-cohort design using survival analysis methods, including log rank statistics and Cox proportional hazards models, were used to quantify differences in incident PC, metastatic PC, Gleason score 8-10 PC, and PC mortality, between exposure groups. Because combination therapy was common, 5ARI users could have also used a-blockers or other drugs of interest, and these were included as covariates in fully-adjusted multivariable models. Exit date (i.e., censoring date) was defined as the earliest of date of cancer diagnosis, coverage termination with SH, death, or December 31, 2014, in that order. Follow-up time comprised the number of days between index and exit date.

For each outcome, two regression models were run: basic models adjusted only for age at index (as a covariate), and multivariable models adjusted for age at index, index year, and baseline use of lipid-lowering medications and cardiometabolic conditions. In sensitivity analyses, Cox proportional hazards models accounting for competing risks were run [21]. As a proxy for drug duration, effect measure modification by index year was evaluated by including an interaction term with drug user group in Cox regression models. Thus, index year was used as a surrogate measure of a drug user's time on drug treatment, with earlier index year representing longer exposure to the drug. Statistical analyses were performed using SAS 9.4 software (SAS Institute, Inc.), with 2-tailed tests and P values $<0.05$ representing statistical significance. The proportional hazards assumption was tested with PROC PHREG.

\section{Results}

On average, $a$-blocker users were younger at index and had a longer median follow-up time than 5ARI users and non-users of either drug (Table 1). 5ARI user and non-user groups had similar amounts of missing data on Gleason score (21\% [56/264] and 22\% [288/1287] within each group, respectively), but more a-blocker users had missing data (36\% [316/882]). Distributions of the number of prescriptions filled among 5ARI users and $a-$ blocker users with at least 2 prescriptions during study follow-up are provided as a supplemental figure (Fig S1). When comparing 5ARI users with non-users using Cox proportional hazards regression, 5ARI use was associated with a $40 \%$ lower relative risk of 
diagnosed PC in age-adjusted analyses, with the multivariable-adjusted relative risk virtually identical (aHR: 0.61, 95\% CI: 0.53-0.70, P<0.0001) (Table 2). Occurrence of metastatic PC at diagnosis and PC mortality were not significantly different between 5ARI users and nonusers $(\mathrm{P}=0.54$ and $\mathrm{P}=0.49$, respectively); however, both endpoints showed a significant interaction with index year, such that 5ARI users with the most recent index years (20102014) had 2.94 times the risk of metastatic PC and 2.22 times the risk of PC mortality compared with non-users, while null associations were observed for earlier index years (Table S1). While there are intrinsic limitations to the use of Gleason score, we did find that among men with Gleason score data, 5ARI use was associated with a 37\% higher risk of Gleason score 8-10 cancer as compared to non-users (aHR:1.37, 95\% CI: 1.03-1.82, $\mathrm{P}=0.03$ ). For all models, the proportional hazards assumption was met, and accounting for competing risks yielded identical results.

Comparing a-blocker users with non-users, a-blocker users had an $11 \%$ lower risk of diagnosed PC (aHR: 0.89, 95\% CI: 0.81-0.97, $\mathrm{P}=0.01$ ), no association with metastatic PC or $\mathrm{PC}$ mortality $(\mathrm{P}=0.35$ and $\mathrm{P}=0.10$, respectively), but a $28 \%$ higher risk of Gleason score 8 10 cancer than non-users (aHR: $1.28,95 \%$ CI: $1.03-1.59, \mathrm{P}=0.02$ ). Furthermore, an interaction between a-blocker use vs. non-use and index year suggested that protection against a PC diagnosis did not exist among men with later index years (2005-2009 or 20102014, Table S1). When 5ARI users were compared with a-blocker users, 5ARI users showed a 37\% lower risk of diagnosed PC (aHR: 0.63, 95\% CI: 0.54-0.73, $\mathrm{P}<0.0001$ ), but no significant differences for other $\mathrm{PC}$ outcomes $(\mathrm{P}=0.54$ for metastatic $\mathrm{PC}, \mathrm{P}=0.98$ for Gleason score $8-10 \mathrm{PC}$, and $\mathrm{P}=0.26$ for $\mathrm{PC}$ mortality, Table 2). Kaplan-Meier curves for diagnosed PC and PC mortality are shown as Fig. 1 and 2.

\section{Discussion}

5ARI therapy is now established as an evidence-based standard of care for the treatment of selected men with BPH [22]. However, despite multiple studies confirming a lower risk of diagnosed PC in men treated with 5ARIs for BPH [23-25], as well as in populations of men considered as low risk [3] and high risk [5] for development of PC, the use of this strategy for chemoprevention of PC has not become standard of care. The primary reasons for this general disregard for 5ARI PC chemoprevention use in clinical practice was the observation in the REDUCE and PCPT studies [3-5] of a higher risk of high-grade (Gleason 8-10) PC in the 5ARI treated group and the absence of data demonstrating that 5ARIs decrease PC mortality. Many re-analyses [4, 6, 20, 26, 27] have shown that this perceived risk of highgrade cancer with 5ARI therapy may be a pathologic artifact; however, despite these considerations, the controversies related to this issue have led to a general reluctance to recommend a 5ARI chemoprevention strategy in men. Physicians who prescribe 5ARI therapy for men with BPH struggle with the dilemma of potentially subjecting their patients to an increased risk of developing high-grade cancer [20] and its consequences. Reassuring long-term follow-up data from PCPT showed no difference in overall survival with the use of finasteride [4].

The present longitudinal, population-based pharmacoepidemiologic analyses using SH data to examine the use of currently prescribed drugs, 5ARIs and a-blockers, in relation to PC 
risk, contributes to the evidence that can be used to counsel $\mathrm{BPH}$ patients considering medical therapy. Among men with a physician claim reporting BPH, we observed a $40 \%$ reduced risk of PC diagnosis among 5ARI (with or without a-blockers) users compared with non-users and a-blocker (alone) users, which was consistent with the literature predictions [3, 5, 24, 25]. Similarly, the hypothesis, based on REDUCE and PCPT findings [3-5], that we would observe more high-grade PC in the 5ARI group, was confirmed with more Gleason score 8-10 cancer in 5ARI users vs. non-users but also in a-blocker users vs. nonusers. Most importantly, the results showed null findings for mortality outcomes. Furthermore, later drug initiation was associated with higher risk of metastatic PC and PC mortality among 5ARI users vs. non-users, while earlier drug initiation showed null associations with these outcomes.

The implications for BPH patients presently being treated with 5ARI therapy and both patients and physicians considering 5ARI therapy for symptomatic BPH are profound. Based on our study, the longitudinal evaluation of some PCPT subjects [4], as well as other epidemiological studies [4, 11, 14-16], we can counsel our patients with "true" BPH (an enlarged prostate) that they can consider the evidence-based benefits of 5ARI therapy without being exposed to a higher risk of dying of PC. Furthermore, they can expect to have a lower risk of actually being diagnosed with PC. The increased risk of having a higher Gleason grade PC identified at time of PC diagnosis, potentially due to 5ARI-induced increased biopsy sensitivity [27], did not translate into an increased risk of dying from PC. In fact, earlier exposure to 5ARI therapy did not show higher risk of metastatic PC and PC mortality, as suggested with the latest exposure (i.e., index years 2010-2014). Men entering the study earlier were likely to be exposed to the drug longer than those entering later. While our data cannot be used for confirmation, it could be hypothesized that actual diagnosis of higher grade cancer at time of diagnosis could presumably lead to more appropriate, potentially curative therapy. Nonetheless, because the causal relationship driving higher Gleason grade PC diagnosis among 5ARI patients has not been determined, a certain level of caution is still warranted. With respect to a-blocker therapy for BPH, the use (or addition) of a-blockers similarly resulted in a reduced risk of PC diagnosis, increased risk of highgrade PC but no significant effect on PC metastatic disease or PC mortality.

From a rich dataset of linked de-identified administrative health claims data, electronic prescription records, and cancer registry data on over 200,000 men, a large subset with a BPH-coded medical claim comprised the present analysis. Moreover, this study's long follow-up time (up to 20 years) is sparse in prior studies $[11,14,16]$. With a large sample and extended follow-up, the study database included relatively large numbers of events for the main outcomes of interest, improving statistical power compared with prior studies. While previous research has been conducted in specific target populations with limited external validity, the study's large community-based sample provides more generalizable findings. Further, because the population under study has universal health care, socioeconomic biases related to access to care may have been diminished.

As with use of all extant datasets, the study had some unavoidable limitations. For example, a necessary assumption was that a dispensed prescription was a consumed prescription. Missing data, including Gleason score among $21-36 \%$ of drug exposure groups, potentially 
informative behavioral information such as body composition measures, dietary intake and/or dietary supplements, other comorbidities, and family history data, posed analytic challenges. Although unavailable in our dataset, race/ethnicity data from the Saskatchewan census [28] suggest a relatively homogeneous non-minority population, which limits generalizability to other racial/ethnic groups. Moreover, we had somewhat limited data on cancer progression and treatment.

\section{Conclusions}

We observed $>30 \%$ reduced risk of PC diagnosis and no difference in PC mortality among 5ARI users compared with non-users and a-blocker users with a BPH-coded medical claim. More advanced PC (e.g., more Gleason score 8-10 cancer) in 5ARI users vs. non-users and a-blocker users vs. non-users merits continued caution and further investigation. The most important message to our BPH patients is that the present study indicated that medical therapy, specifically 5ARI therapy (and particularly those taking these drugs for longer periods of time), is associated with a potential of reduced risk of being diagnosed with PC that does not appreciably change the risk of developing metastatic PC or dying of PC. Our data cannot be used to justify a PC chemoprevention strategy for men with or without BPH.

\section{Supplementary Material}

Refer to Web version on PubMed Central for supplementary material.

\section{Acknowledgements}

This work was supported by the National Institutes of Health National Institute on Aging under Award Number R01AG038453. The National Institute on Aging played no direct role in the study. The content is solely the responsibility of the authors and does not necessarily represent the official views of the National Institutes of Health. This study is based in part on de-identified data provided by the Saskatchewan Ministry of Health. The interpretation and conclusions contained herein do not necessarily represent those of the Government of Saskatchewan or the Saskatchewan Ministry of Health. The authors gratefully acknowledge the support of MaryRose Stang of the Population Health Branch, Saskatchewan Ministry of Health, and Michael Maurao, Weiqin Liao, and Ellen Cho of HealthCore/NERI.

\section{Abbreviations:}

$\begin{array}{ll}\text { 5ARI } & \text { 5a-reductase inhibitor } \\ \text { aHR } & \text { adjusted hazard ratio } \\ \text { BPH } & \text { benign prostatic hyperplasia } \\ \text { PC } & \text { prostate cancer } \\ \text { PCPT } & \text { Prostate Cancer Prevention Trial } \\ \text { REDUCE } & \text { Reduction by Dutasteride of Prostate Cancer Events } \\ \text { SCA } & \text { Saskatchewan Cancer Agency } \\ \text { SH } & \text { Saskatchewan Health }\end{array}$




\section{References}

1. Cancer Stat Facts: Prostate Cancer [Internet]. 2017 Available from: https://seer.cancer.gov/statfacts/ html/prost.html.

2. Sandhu GS, Nepple KG, Tanagho YS, Andriole GL. Prostate cancer chemoprevention. Semin Oncol 2013; 40: 276-85 [PubMed: 23806493]

3. Thompson IM, Goodman PJ, Tangen CM, et al. The influence of finasteride on the development of prostate cancer. N Engl J Med 2003; 349: 215-24 [PubMed: 12824459]

4. Thompson IM, Jr., Goodman PJ, Tangen CM, et al. Long-term survival of participants in the prostate cancer prevention trial. N Engl J Med 2013; 369: 603-10 [PubMed: 23944298]

5. Andriole GL, Bostwick DG, Brawley OW, et al. Effect of dutasteride on the risk of prostate cancer. N Engl J Med 2010; 362: 1192-202 [PubMed: 20357281]

6. Grubb RL, Andriole GL, Somerville MC, Mahoney C, Manyak MJ, Castro R. The REDUCE Follow-Up Study: low rate of new prostate cancer diagnoses observed during a 2-year, observational, followup study of men who participated in the REDUCE trial. J Urol 2013; 189 : 871-7 [PubMed: 23021996]

7. Wilt TJ, Macdonald R, Hagerty K, et al. 5-alpha-Reductase inhibitors for prostate cancer chemoprevention: an updated Cochrane systematic review. BJU Int 2010; 106: 1444-51 [PubMed: 20977593]

8. Food US and Administration Drug. FDA Drug Safety Communication: 5-alpha reductase inhibitors (5-ARIs) may increase the risk of a more serious form of prostate cancer2011. Available from: https://www.fda.gov/Drugs/DrugSafety/ucm258314.htm. Accessed on 3/29/2017.

9. Roehrborn CG, Andriole GL, Wilson TH, Castro R, Rittmaster RS. Effect of dutasteride on prostate biopsy rates and the diagnosis of prostate cancer in men with lower urinary tract symptoms and enlarged prostates in the Combination of Avodart and Tamsulosin trial. Eur Urol 2011; 59: 244-9 [PubMed: 21093145]

10. Monga N, Sayani A, Rubinger DA, Wilson TH, Su Z. The effect of dutasteride on the detection of prostate cancer: A set of meta-analyses. Can Urol Assoc J 2013; 7: E161-7 [PubMed: 23589750]

11. Preston MA, Wilson KM, Markt SC, et al. 5alpha-Reductase inhibitors and risk of high-grade or lethal prostate cancer. JAMA Intern Med 2014; 174: 1301-7 [PubMed: 24887392]

12. Murtola TJ, Tammela TL, Maattanen L, Ala-Opas M, Stenman UH, Auvinen A. Prostate cancer incidence among finasteride and alpha-blocker users in the Finnish Prostate Cancer Screening Trial. Br J Cancer 2009; 101: 843-8 [PubMed: 19654575]

13. Orsted DD, Bojesen SE, Nielsen SF, Nordestgaard BG. Association of clinical benign prostate hyperplasia with prostate cancer incidence and mortality revisited: a nationwide cohort study of 3,009,258 men. Eur Urol 2011; 60: 691-8 [PubMed: 21705134]

14. Azoulay L, Eberg M, Benayoun S, Pollak M. 5alpha-Reductase Inhibitors and the Risk of CancerRelated Mortality in Men With Prostate Cancer. JAMA Oncol 2015; 1: 314-20 [PubMed: 26181177]

15. Wallner LP, DiBello JR, Li BH, et al. 5-Alpha Reductase Inhibitors and the Risk of Prostate Cancer Mortality in Men Treated for Benign Prostatic Hyperplasia. Mayo Clin Proc 2016; 91: 1717-26 [PubMed: 28126151]

16. Murtola TJ, Karppa EK, Taari K, Talala K, Tammela TL, Auvinen A. 5-Alpha reductase inhibitor use and prostate cancer survival in the Finnish Prostate Cancer Screening Trial. Int J Cancer 2016; 138: 2820-8 [PubMed: 26804670]

17. Hagberg KW, Divan HA, Persson R, Nickel JC, Jick SS. Risk of erectile dysfunction associated with use of 5-alpha reductase inhibitors for benign prostatic hyperplasia or alopecia: population based studies using the Clinical Practice Research Datalink. BMJ 2016; 354: i4823 [PubMed: 27659058]

18. Delahunt B, Egevad L, Grignon DJ, Srigley JR, Samaratunga H. Prostate cancer grading: recent developments and future directions. BJU Int 2016; 117 Suppl 4: 7-8

19. Albertsen PC, Hanley JA, Barrows GH, et al. Prostate cancer and the Will Rogers phenomenon. J Natl Cancer Inst 2005; 97: 1248-53 [PubMed: 16145045] 
20. Thompson IM, Klein EA, Lippman SM, Coltman CA, Djavan B. Prevention of prostate cancer with finasteride: US/European perspective. Eur Urol 2003; 44: 650-5 [PubMed: 14644115]

21. Kohl M, Plischke M, Leffondre K, Heinze G. PSHREG: a SAS macro for proportional and nonproportional subdistribution hazards regression. Comput Methods Programs Biomed 2015; 118: 218-33 [PubMed: 25572709]

22. American Urological Association. Management of Benign Prostatic Hyperplasia (BPH)2014. Available from: http://www.auanet.org/guidelines/benign-prostatic-hyperplasia-(2010-reviewedand-validity-confirmed-2014).

23. Andriole GL, Guess HA, Epstein JI, et al. Treatment with finasteride preserves usefulness of prostate-specific antigen in the detection of prostate cancer: results of a randomized, double-blind, placebo-controlled clinical trial. PLESS Study Group. Proscar Long-term Efficacy and Safety Study. Urology 1998; 52: 195-201; discussion -2 [PubMed: 9697781]

24. Roehrborn CG, Siami P, Barkin J, et al. The effects of combination therapy with dutasteride and tamsulosin on clinical outcomes in men with symptomatic benign prostatic hyperplasia: 4-year results from the CombAT study. Eur Urol 2010; 57: 123-31 [PubMed: 19825505]

25. Andriole GL, Roehrborn C, Schulman C, Slawin KM, Somerville M, Rittmaster RS. Effect of dutasteride on the detection of prostate cancer in men with benign prostatic hyperplasia. Urology 2004; 64: 537-41; discussion 42-3 [PubMed: 15351586]

26. Lacy JM, Kyprianou N. A tale of two trials: The impact of 5alpha-reductase inhibition on prostate cancer (Review). Oncol Lett 2014; 8: 1391-6 [PubMed: 25202340]

27. Redman MW, Tangen CM, Goodman PJ, Lucia MS, Coltman CA, Jr., Thompson IM. Finasteride does not increase the risk of high-grade prostate cancer: a bias-adjusted modeling approach. Cancer Prev Res (Phila) 2008; 1: 174-81 [PubMed: 19138953]

28. Statistics Canada. 2006 Community Profiles. 2006 Census. Statistics Canada Catalogue no 92 591-XWE [Internet]. 2007 Available from: http://www12.statcan.ca/census-recensement/2006/dppd/prof/92-591/index.cfm?Lang=E. Accessed on 8/8/2018. 
(A) 5ARI Users vs. Non-users

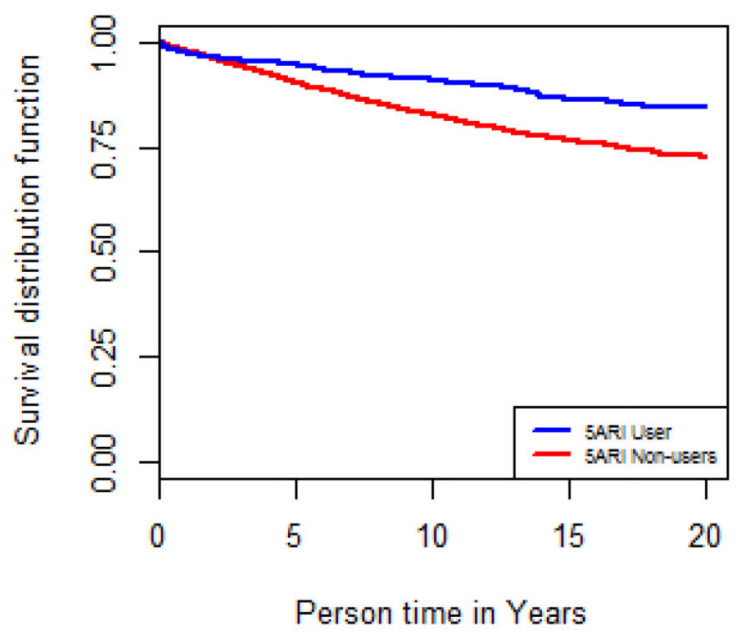

(C) 5ARI Users vs. Alpha-Blocker Users

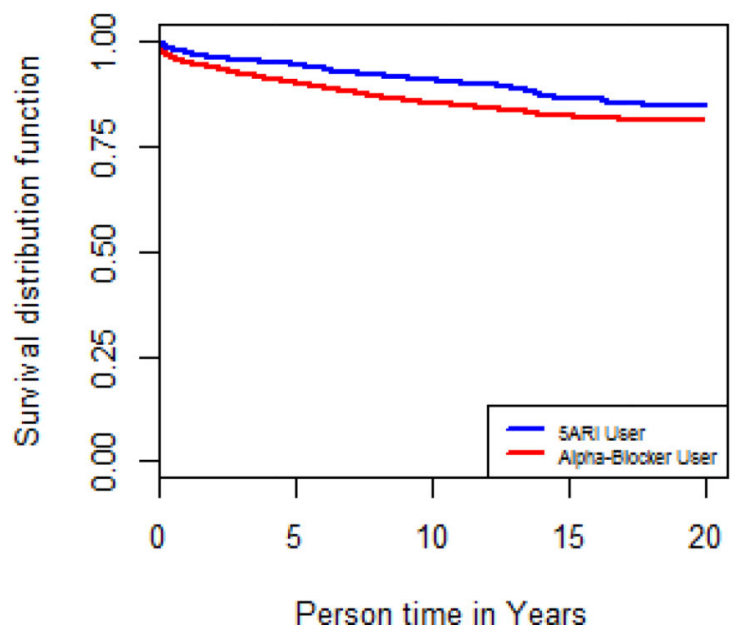

(B) Alpha-Blocker Users vs. Non-users

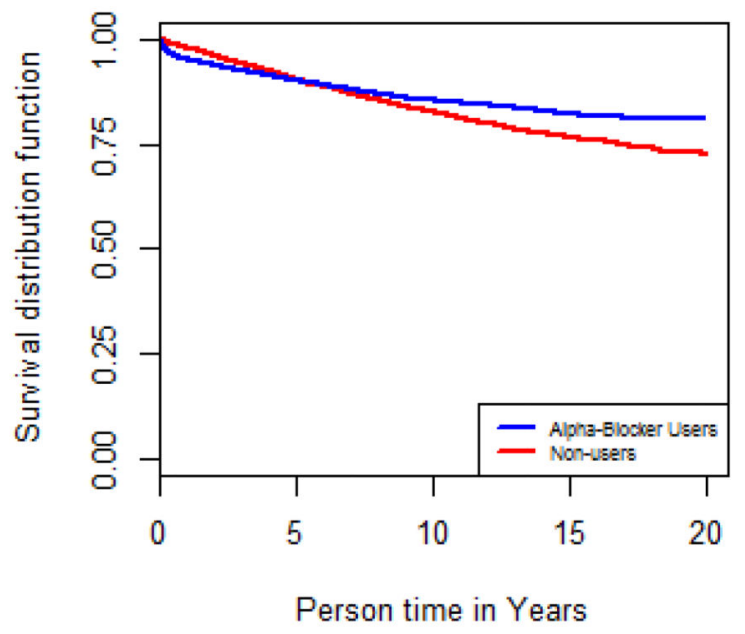

Fig. 1.

Multivariable-adjusted Kaplan-Meier curves for prostate cancer incidence among male new users of 5a-reductase inhibitors (5ARI, $n=4,571)$, a-blockers $(n=7,764)$, and non-users $(11,677)$ with a BPH-coded medical claim. Kaplan-Meier curves and hazard ratios were adjusted for age at index, index year, baseline cardiometabolic conditions (diabetes, hypertension, heart disease, and/or hyperlipidemia), and baseline use of lipid-lowering drugs (statins and/or non-statin lipid-lowering medications): (A) 5ARI users vs. non-users HR $\mathbf{0 . 6 1}$ (95\% CI: 0.53-0.70), P<0.0001; (B) a-blocker users vs. non-users HR 0.89 (95\% CI: 0.810.97), $\mathrm{P}=0.01$; (C) 5ARI users vs. a-blocker users HR 0.63 (95\% CI: 0.54-0.73), $\mathrm{P}<0.0001$. 
(A) 5ARI Users vs. Non-users

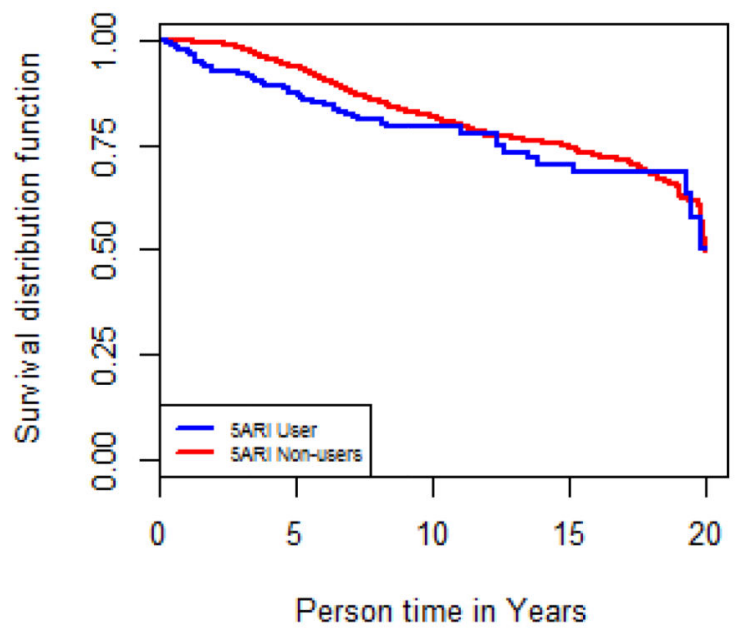

(C) 5ARI Users vs. Alpha-Blocker Users

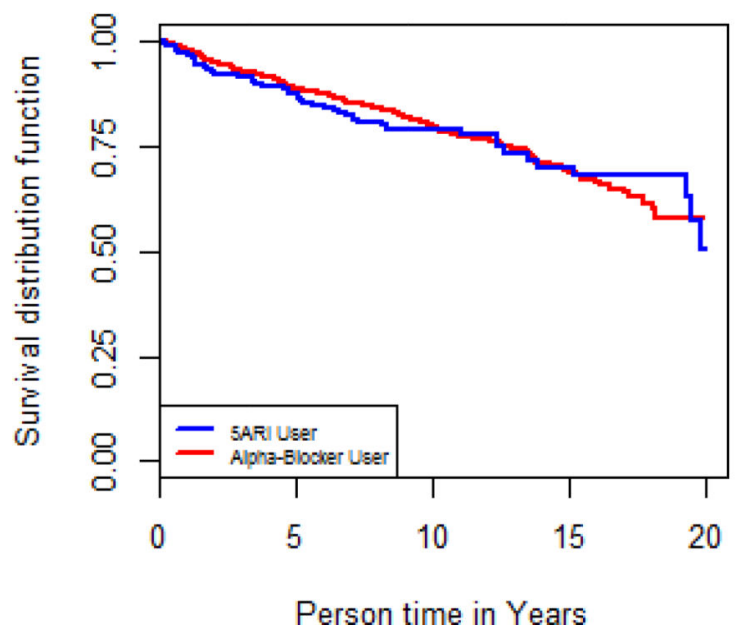

(B) Alpha-Blocker Users vs. Non-users

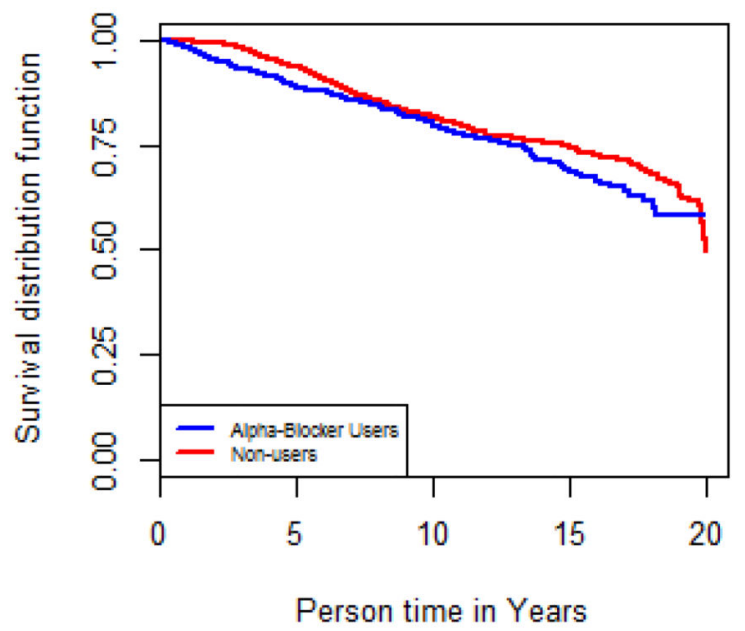

Fig. 2.

Multivariable-adjusted Kaplan-Meier curves for prostate cancer mortality among male new users of 5a-reductase inhibitors (5ARI, $n=4,571)$, $a$-blockers $(n=7,764)$, and non-users $(11,677)$ with a BPH-coded medical claim. Kaplan-Meier curves and hazard ratios were adjusted for age at index, index year, baseline cardiometabolic conditions (diabetes, hypertension, heart disease, and/or hyperlipidemia), and baseline use of lipid-lowering drugs (statins and/or non-statin lipid-lowering medications): (A) 5ARI users vs. non-users HR $\mathbf{1 . 1 1}$ (95\% CI: 0.82-1.50), P=0.49; (B) a-blocker users vs. non-users HR 1.18 (95\% CI: $0.97-$ 1.44) $\mathrm{P}=0.10$; (C) 5ARI users vs. a-blocker users HR $\mathbf{0 . 8 4}$ (95\% CI: 0.61-1.14), $\mathrm{P}=0.26$. 
Table 1.

Characteristics of 5a-reductase inhibitor (5ARI) users, a-blocker users, and non-users with a BPH-coded medical claim

\begin{tabular}{|c|c|c|c|}
\hline & 5ARI users* & a-blocker users & Non-users \\
\hline $\mathbf{N}$ & 4571 & 7764 & 11677 \\
\hline Age at index (yrs), Mean (SD) & $70.0(9.3)$ & $66.7(9.7)$ & $71.0(8.5)$ \\
\hline \multicolumn{4}{|l|}{ Year of index, $\mathrm{n}(\%)$} \\
\hline 1995-2004 & $964(21.1 \%)$ & $4011(51.7 \%)$ & $3913(33.5 \%)$ \\
\hline 2005-2009 & $1367(29.9 \%)$ & $1941(25.0 \%)$ & $3408(29.2 \%)$ \\
\hline 2010-2014 & $2240(49.0 \%)$ & $1812(23.3 \%)$ & $4356(37.3 \%)$ \\
\hline \multicolumn{4}{|l|}{ Follow-up time (yrs) } \\
\hline Median (IQR) & $4.1(5.1)$ & $6.3(8.0)$ & $5.0(6.2)$ \\
\hline Min-Max & $0.003-20$ & $0.003-20$ & $0.003-20$ \\
\hline Age at exit (yrs), Mean (SD) & $74.9(9.4)$ & $73.7(10.1)$ & $77.1(8.1)$ \\
\hline Baseline lipid-lowering drug use, $n(\%)^{\dagger}$ & $1879(41.1 \%)$ & $2242(28.9 \%)$ & $4469(38.3 \%)$ \\
\hline Baseline cardiometabolic conditions, $\mathbf{n}(\%)^{*}$ & $3790(82.9 \%)$ & $5658(72.9 \%)$ & $9593(82.1 \%)$ \\
\hline \multicolumn{4}{|l|}{ Outcomes during follow-up, n (\%) } \\
\hline Prostate cancer & $264(5.8 \%)$ & $882(11.4 \%)$ & $1287(11.0 \%)$ \\
\hline \multicolumn{4}{|l|}{ Advanced prostate cancer } \\
\hline Metastases at diagnosis & $37(0.8 \%)$ & $113(1.5 \%)$ & $162(1.4 \%)$ \\
\hline Gleason score $8-10$ & $66(1.4 \%)$ & $139(1.8 \%)$ & $232(2.0 \%)$ \\
\hline Complete Gleason score data & $208(78.8 \%)$ & $566(64.2 \%)$ & $999(77.6 \%)$ \\
\hline \multicolumn{4}{|l|}{ Mortality among men with PC } \\
\hline Prostate cancer & $53(1.2 \%)$ & $188(2.4 \%)$ & $257(2.2 \%)$ \\
\hline Other cancer & $16(0.4 \%)$ & $48(0.6 \%)$ & $72(0.6 \%)$ \\
\hline Non-cancer & $41(0.9 \%)$ & $186(2.4 \%)$ & $236(2.0 \%)$ \\
\hline Missing & $154(3.4 \%)$ & $460(5.9 \%)$ & $722(6.2 \%)$ \\
\hline
\end{tabular}


Table 2.

Risk of prostate cancer outcomes among 5a-reductase inhibitor users (5ARI, $\mathrm{n}=4,571$ ), $\mathrm{a}$-blocker users $(\mathrm{n}=7,764)$, and non-users $(\mathrm{n}=11,677)$ with a BPH-coded medical claim

\begin{tabular}{|c|c|c|c|c|c|}
\hline & No. of events & $\begin{array}{c}\text { Age-adjusted HR } \\
\text { (95\% CI) }\end{array}$ & $P$ value & Fully-adjusted HR $(95 \% \mathrm{CI})^{*}$ & $P$ value \\
\hline \multicolumn{6}{|l|}{ 5ARI users ${ }^{\dagger}$ vs. non-users } \\
\hline Total prostate cancer & 1551 & $0.60(0.52-0.68)$ & $<0.0001$ & $0.61(0.53-0.70)$ & $<0.0001$ \\
\hline Metastatic prostate cancer & 199 & $1.13(0.79-1.62)$ & 0.50 & $1.12(0.78-1.61)$ & 0.54 \\
\hline Gleason score 8-10 cancer & 298 & $1.51(1.14-2.00)$ & 0.005 & $1.37(1.03-1.82)$ & 0.03 \\
\hline Prostate cancer mortality & 310 & $1.13(0.83-1.53)$ & 0.44 & $1.11(0.82-1.50)$ & 0.49 \\
\hline \multicolumn{6}{|l|}{ a-blocker users vs. non-users } \\
\hline Total prostate cancer & 2169 & $0.94(0.86-1.03)$ & 0.21 & $0.89(0.81-0.97)$ & 0.01 \\
\hline Metastatic prostate cancer & 275 & $1.04(0.82-1.33)$ & 0.75 & $1.13(0.88-1.45)$ & 0.35 \\
\hline Gleason score $8-10$ cancer & 371 & $1.14(0.93-1.42)$ & 0.21 & $1.28(1.03-1.59)$ & 0.02 \\
\hline Prostate cancer mortality & 445 & $1.14(0.94-1.38)$ & 0.19 & $1.18(0.97-1.44)$ & 0.10 \\
\hline \multicolumn{6}{|l|}{ 5ARI users vs. $a$-blocker users } \\
\hline Total prostate cancer & 1146 & $0.56(0.49-0.65)$ & $<0.0001$ & $0.63(0.54-0.73)$ & $<0.0001$ \\
\hline Metastatic prostate cancer & 150 & $1.09(0.76-1.57)$ & 0.63 & $0.89(0.61-1.30)$ & 0.54 \\
\hline Gleason score 8-10 cancer & 205 & $1.32(0.98-1.76)$ & 0.06 & $1.00(0.75-1.35)$ & 0.98 \\
\hline Prostate cancer mortality & 241 & $0.94(0.69-1.28)$ & 0.69 & $0.84(0.61-1.14)$ & 0.26 \\
\hline
\end{tabular}

* HR adjusted for age at index, index year, baseline use of lipid-lowering drugs (statins and/or non-statin lipid-lowering medications), and baseline cardiometabolic conditions (diagnosed diabetes, hypertension, heart disease, and/or hyperlipidemia).

t”,5ARI users" could have also used a-blockers, but “a-blocker users" used a-blockers only 